\title{
On Symmetric Riemann-Derivatives
}

\section{S. Deb}

Abstract: The basic properties like monotoni city, Darboux property, mean value property of symmetric Riemann-derivatives of order $n$ of a real valued function $f$ at a point $x$ of its domain (a closed interval) is studied. In some cases, function is considered to be continuous or semi-continuous.

Keywords: Symmetric derivatives, Riemann-derivatives, symmetric Riemann-derivatives. Mathematics Subject Classification (2020): 26E99, $28 E 99$.

\section{INTRODUCTION}

$S_{\text {ymmetric Riemann-derivatives is a generalization of }}$ normal or ordinary or general symmetric derivatives. It is a blender of Riemann-derivatives and symmetric derivatives to form a new generalization of derivatives. Mathematicians worked towards Symmetric derivatives and Riemann-derivatives, then got a new outlook towards symmetric Riemann-derivatives. In 1954, P. L. Butzer and W. Kozakiewicz showed their work on the Riemannderivatives for integrable functions [7]. Later J. Marshall Ash, Stefan Catoiu and William Chin William worked on generalization of Riemann-derivatives and classification of generalized Riemann-derivatives(1967) [1] [2]. In 1974, P. S. Bullen and S. N. Mukhopadhyay discovered relation between different generalized derivatives [6]. From 1970 and 1974, N. K. Kundu researched on properties of symmetric derivatives including conditions on a function's symmetric derivatives for monotonicity [10] [11]. Around 1972, C. L. Belna , M. J. Evans and P. D. Humke , on symmetric and ordinary differentiation [3]. Sorin Radulescu, Petrus Alexandrescu and Diana-Olimpia Alexandrescu published their paper on generalized Riemann-derivatives and it's reference to study of qualitative property of a function in 2013 [13] [14]. Subhankar Ghosh worked on same field in his Ph. D. Thesis, namely SOME STUDIES ON HIGHER ORDER GENERALIZED DERIVATIVES, SYMMETRIC DERIVATIVES, DIVIDED DIFFERENCES AND THEIR INTERRELATIONS to Visva-Bharati University in 2017 [8]. Many mathematicians such as B. S. Thompson [15], R. G. Bertle and D. R. Sherbert [4], A. Zygmund [16], A. Gordon Russel [9], S. N. Mukhopadhyay [12], A. M. Bruckner [5] compiled the findings so far in books or papers along with something new. In this section we have studied $n^{\text {th }}$ order symmetric Riemann-derivatives and have shown by example that symmetric Riemann-derivatives is more general than symmetric derivative.

Manuscript received on 29 September 2021 | Revised Manuscript received on 09 October 2021 | Manuscript Accepted on 15 October 2021 | Manuscript published on 30 October 2021.

* Correspondence Author

S. Deb*, Student, Department of Mathematics, Visva-Bharati, Santiniketan, Bolpur (West Bengal), India. E-mail: debsuranjana@gmail.com

(C) The Authors. Published by Lattice Science Publication (LSP). This is an open access article under the CC-BY-NC-ND license (http://creativecommons.org/licenses/by-nc-nd/4.0/)
Also we have proved some theorems regarding monotonicity and mean value theorem for symmetric Riemann-derivatives of a function having upper semicontinuity and with property D as well as it's relation with Riemann-derivatives.

\section{DEFINITIONS AND NOTATIONS}

Definition 2.1. Let $f \quad: \mathbf{R} \rightarrow \mathbf{R}$ be a function. If $\quad \limsup _{h \rightarrow 0} \frac{\Delta_{n}^{s}(f, x, h)}{h^{n}}$ exists, where $\Delta_{n} s(f, x, h)$ $=\sum_{i=0}^{n}(-1)^{n-i}\left(\begin{array}{l}n \\ i\end{array}\right) f\left(x-\frac{n h}{2}+i h\right)$, then this limit is said to be the n-th upper symmetric Riemann-derivative of $f$ at $x$ and is denoted by $S_{R} D_{n}(x)$. Similarly, the limit $\liminf _{h \rightarrow 0} \frac{\Delta_{n}^{s}(f, x, h)}{h^{n}}$, if exists, is said to be the $n$-th lower symmetric Riemann-derivative of $f$ at $x$ and is denoted by $\underline{S R D_{n} f(x)}$.

If both $S R D_{n}^{+} f(x)$ and ${\underline{S R D_{n}}}^{-} f(x)$ exist and are equal, then this common value is said to be the n-th symmetric Riemann-derivative of $f$ at $x$ and is denoted by $S R D_{n} f$ $(x)$.

Example 2.2. (i) Let $f(x)=e^{x}$.

$$
\begin{array}{r}
\Delta_{n}^{s}(f, x, t)=\sum_{i=0}^{n} \quad(-1)^{n-i}\left(\begin{array}{c}
n \\
i
\end{array}\right) f\left(x-\frac{n t}{2}+i t\right) \\
=\sum_{i=0}^{n} \quad(-1)^{n-i}\left(\begin{array}{c}
n \\
i
\end{array}\right) e^{x-\frac{n i t}{2}+i t} \\
=e^{x} \sum_{i=0}^{n} \quad(-1)^{n-i}\left(\begin{array}{c}
n \\
i
\end{array}\right) e^{(i-n) t} \\
\frac{\Delta_{n}^{s}(f, x, t)}{t^{n}}=e^{x} \sum_{i=0}^{n} \quad(-1)^{n-i}\left(\begin{array}{l}
n \\
i
\end{array}\right) \frac{e^{(i-n) t}}{t^{n}}
\end{array}
$$

(ii) Let $f(x)=\sin x$.

$$
\begin{gathered}
\Delta_{n}^{s}(f, x, t)=\sum_{i=0}^{n} \quad(-1)^{n-i}\left(\begin{array}{c}
n \\
i
\end{array}\right) f\left(x-\frac{n t}{2}+i t\right) \\
=\sum_{i=0}^{n} \quad(-1)^{n-i}\left(\begin{array}{c}
n \\
i
\end{array}\right) \sin \left\{x-\frac{n t}{2}+i t\right\}
\end{gathered}
$$




\section{On Symmetric Riemann-Derivatives}

$$
=\sum_{i=0}^{n} \quad(-1)^{n-i}\left(\begin{array}{l}
n \\
i
\end{array}\right)[\sin x \cos (i-n) t+\cos x \sin (i-n) t]
$$

$\lim _{t \rightarrow 0} \frac{\Delta_{n}^{s}(f, x, t)}{t^{n}}=\sin x \sum_{i=0}^{n}(-1)^{n-i}\left(\begin{array}{l}n \\ i\end{array}\right)\left[\lim _{t \rightarrow 0+} \frac{\cos (i-n) t}{t^{n}}\right]+\cos x \sum_{i=0}^{n} \quad(-1)^{n-i}\left(\begin{array}{l}n \\ i\end{array}\right)\left[\lim _{t \rightarrow 0+} \frac{\sin (i-n) t}{t^{n}}\right]$

(iii) Let $f(x)=x^{2}$.

$$
\begin{gathered}
\Delta_{1}^{s}(f, x, t)=f\left(x+\frac{t}{2}\right)-f\left(x-\frac{t}{2}\right)=\left(x+\frac{t}{2}\right)^{2}-\left(x-\frac{t}{2}\right)^{2}=4 x^{\frac{t}{2}}=2 x t \\
\Delta_{2}^{s}(f, x, t)=\Delta^{s} f\left(x+\frac{t}{2}\right)-\Delta^{s} f\left(x-\frac{t}{2}\right) \\
=f(x+t)-2 f(x)+f(x-t) \\
=(x+t)^{2}-2(x)^{2}+(x-t)^{2} \\
=2 t^{2}
\end{gathered}
$$$$
\Delta_{n}^{s}(f, x, t)=0 \text { if } n>2 .
$$

So, $S R D_{1} f(x)=2 x=f^{\prime}(x)=R D_{1} f(x), S R D_{2} f(x)=2=f^{\prime \prime}(x)=R D_{2} f(x)$.

Note 2.3. Let

$$
\begin{gathered}
f(x)=x^{2} \sin ^{\frac{1}{x}} \text { when } x \in Q \\
=x^{3} \text { when } x \in Q
\end{gathered}
$$

Then $f^{\prime \prime}(0), f_{2}(0), S D f^{2}(0)$ do not exist. But

$S R D_{2} f(0)$

$=\lim _{t \rightarrow 0+} \frac{f(2 t)-2 f(t)+f(0)}{t^{2}}=\lim _{t \rightarrow 0+} \frac{8 t^{3}-2 t^{3}}{t^{2}}=\lim _{t \rightarrow 0+} \frac{6 t^{3}}{t^{2}}=6 \lim _{t \rightarrow 0+} \frac{t^{3}}{t^{2}}=6 \lim _{t \rightarrow 0+} \quad t=0$

So, symmetric Riemann-derivative is more general than ordinary derivative, Peano derivative, symmetric derivative.

\section{SOME RESULTS}

Theorem 3.1. Let $f$ be a continuous real valued function in $[a, b], S R D_{1}^{+} f$ and $S R D_{1}^{-} f$ exist in a set $E$ contained in $[a, b]$, then $S R D_{1}^{+} f$, $S R D_{1}-f \in B_{1}(E)$. Moreover, if (i) $S R D_{n} f$ is finite, (ii) $S R D_{i} f$ is continuous in $E, i=0,1, \ldots, n$, (iii) $S R D_{n+1}^{+} f$ and $S R D_{n+1}-f$ exist in $E$, then $S R D_{n+1}^{+} f, S R D_{n+1}{ }^{-} f \in B_{1}(E)$.

So, $S R D_{1}{ }^{+} f(x) \in B_{1}(E)$.

$$
\lim _{n \rightarrow \infty} \lim _{n \rightarrow \infty} F_{n}(x)=\lim _{h \rightarrow 0+} \frac{f\left(x+\frac{h}{2}\right)-f\left(x-\frac{h}{2}\right)}{h}=S R D_{1}^{+} f(x)
$$

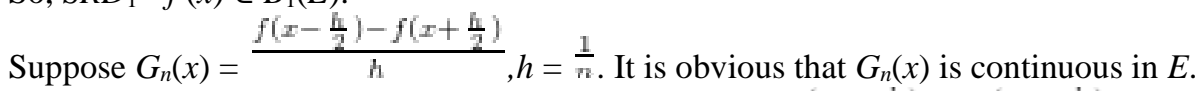

So, $S R D_{1}^{-} f(x) \in B_{1}(E)$.

$$
\lim _{n \rightarrow \infty} G_{n}(x)=\lim _{h \rightarrow 0-} \frac{f\left(x-\frac{h}{2}\right)-f\left(x+\frac{h}{2}\right)}{h}=S R D_{1}-f(x)
$$

Suppose, moreover, if (i) $S R D_{n} f$ is finite, (ii) $S R D_{i} f$ is continuous in E, $i=0,1, \ldots, n$, (iii) $S R D_{n+1}^{+} f$ and $S R D_{n+1}{ }^{-} f$ exist in $E$. Suppose $\Phi_{m}(x)=\frac{\Delta_{n+1}^{n}(f, x, h)}{h^{n+1}}, h=\frac{1}{m}$. It is obvious that $\Phi_{m}(x)$ is continuous in $E$.

So, $S R D_{n+1}{ }^{+} f(x) \in B_{1}(E)$.

$$
\lim _{m \rightarrow \infty} \Phi_{m}(x)=\lim _{h \rightarrow 0} \frac{\Delta_{n+1}^{s}(f, x, h)}{h}=S R D_{n+1}^{+} f(x)
$$

Suppose $\Psi_{m}(x)=\frac{\Delta_{n+1}^{n}(f, x,-h)}{(-h)^{n+1}}, h=\frac{1}{m}$. It is obvious that $\Psi_{n}(x)$ is continuous in $E$.

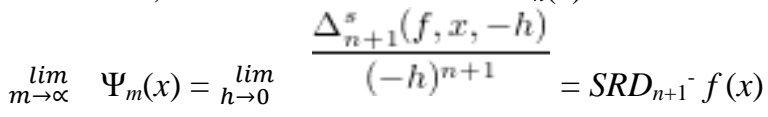

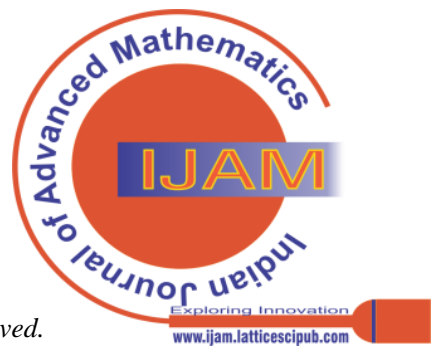


So, $S R D_{n+1}-f(x) \in B_{1}(E)$.

Note 3.2. Let $f$ be a function in $[a, b]$. If $f$ is non-decreasing in $[a, b]$, then $S R D_{n} f(x) \geq 0$ in $[a, b]$.

Proof. Suppose $\alpha, \beta \in[a, b]$, such that $\alpha<\beta$. So, $f(\alpha) \leq f(\beta)$.

Now, for any $x_{0} \in(a, b)$ and for any $\delta$ satisfying $0<\delta<\left(b-x_{0}\right)$, we have $f\left(x_{0}-\frac{n h}{2}+\delta\right) \geq f\left(x_{0}-\frac{n h}{2}\right)$.

$$
\Delta_{n}{ }^{s}(f, x, h)=\sum_{i=0}^{n} \quad(-1)^{n-i}\left(\begin{array}{c}
n \\
i
\end{array}\right) f\left(x-\frac{n h}{2}+i h\right)
$$

Let us take $h(>0)$ in a way such that $\max \{0, h, 2 h, \ldots,(n-1) h\} \leq \delta$.

Hence,

$$
\begin{gathered}
\Delta_{n}^{s}\left(f, x_{0}, h\right)=\sum_{i=0}^{n} \quad(-1)^{n-i}\left(\begin{array}{l}
n \\
i
\end{array}\right) f\left(x_{0}-\frac{n h}{2}+i h\right) \\
\Rightarrow \Delta_{n}^{s}\left(f, x_{0}, h\right) \geq \sum_{i=0}^{n-1} \quad(-1)^{n-i}\left(\begin{array}{c}
n \\
i
\end{array}\right) f\left(x_{0}-\frac{n h}{2}\right)+f\left(x_{0}-\frac{n h}{2}+n h\right) \\
\Rightarrow \Delta_{n}^{s}\left(f, x_{0}, h\right) \geq f\left(x_{0}\right) \sum_{i=0}^{n} \quad(-1)^{n-i}\left(\begin{array}{l}
n \\
i
\end{array}\right)+f\left(x_{0}-\frac{n h}{2}+n h\right)-f\left(x_{0}-\frac{n h}{2}\right) \\
\Rightarrow \Delta_{n}^{s}\left(f, x_{0}, h\right) \geq f\left(x_{0}-\frac{n h}{2}\right)(-1+1)^{n}+f\left(x_{0}-\frac{n h}{2}+n h\right)-f\left(x_{0}-\frac{n h}{2}\right) \\
\Rightarrow \Delta_{n}^{s}\left(f, x_{0}, h\right) \geq f\left(x_{0}-\frac{n h}{2}+n h\right)-f\left(x_{0}-\frac{n h}{2}\right) \geq 0 \\
\text { Then } S R D_{n} f(x)=\lim _{h \rightarrow 0+} \frac{\Delta_{n}^{s}(f, x, h)}{h^{n}} \geq 0, \text { provided the limit exists. }
\end{gathered}
$$

Theorem 3.3. Let $f$ be an upper semi-continuous function which has the property $D$ in $[a, b]$. If $E=\{$ $\left.x \in[a, b]: S R D_{n}^{+} f(x) f \leq 0\right\}$ and $f(E)$ has no subinterval, then $f$ is non-decreasing in $[a, b]$. $f$

Proof. Suppose $\alpha, \beta \in[a, b]$, such that $\alpha<\beta$. So, $f(\alpha)>$

Now, let $y_{0} \in(f(\alpha), f(\beta))$ such that $y_{0}$ doesn't belong to $f$ $(E)$.

Let $S=\left\{x \in[a, b]: f(x) \geq y_{0}\right\}$ and $x_{0}=$
Since $f$ is an upper semi-continuous function with property $D$ in $[a, b], S$ is closed and thus $x_{0} \in S$. Therefore, $f$ $\left(x_{0}\right) \geq y_{0}$. We will show that $f\left(x_{0}\right)=y_{0}$. If not, there exist $\eta$ satisfying $f(\beta)<y_{0}<\eta<f\left(x_{0}\right)$ and $\xi \in$ $\left(x_{0}, \beta\right)$, such that $f(\xi)=\eta$. It contradicts that $x_{0}=\sup S$. So, $f \quad\left(x_{0}\right) \quad=y_{0}$. Since $f$ is an upper semi-continuous function with property $D$ in $[a, b]$ and $x_{0}<\beta$,

If $0<\delta<\left(\beta-x_{0}\right)$, then $f\left(x_{0}+\delta\right)-f\left(x_{0}\right)<0$.

$$
\text { for } x_{0}<x<\beta, f(x)<f\left(x_{0}\right) \text {. }
$$

Again, $f$ being upper semi-continuous function with property $D$ in $[a, b]$, for any $y_{0}>y$ there is a neighbourhood $U$ of $x_{0}$ such that $y<f(x)<y_{0}$, whenever $x \in U$.

Let us take $h(>0)$ in a way such that

$$
\Delta_{n}{ }^{s}\left(f, x_{0}, h\right)=\sum_{i=0}^{n} \quad(-1)^{n-i}\left(\begin{array}{l}
n \\
i
\end{array}\right) f\left(x_{0}-\frac{n h}{2}+i h\right)
$$

$$
\begin{gathered}
x_{0}-\frac{n h}{2}+i h \in U x_{0+} \frac{n h}{2} \text { for all } i=0,1, \ldots, n \text { and } \\
\max \{0, h, 2 h, \ldots,(n-1) h\} \leq \delta .
\end{gathered}
$$

Therefore,

$$
\begin{gathered}
\Delta_{n}{ }^{s}\left(f, x_{0}, h\right)=\sum_{i=0}^{n} \quad(-1)^{n-i}\left(\begin{array}{c}
n \\
i
\end{array}\right) f\left(x_{0}-\frac{n h}{2}+i h\right) \\
\Rightarrow \Delta_{n}{ }^{s}\left(f, x_{0}, h\right)<\sum_{i=0}^{n-1} \quad(-1)^{n-i}\left(\begin{array}{c}
n \\
i
\end{array}\right) f\left(x_{0}-\frac{n h}{2}\right)+f\left(x_{0}+\frac{n h}{2}\right) \\
\Rightarrow \Delta_{n}^{s}\left(f, x_{0}, h\right)<f\left(x_{0}^{\frac{n h}{2}}\right) \sum_{i=0}^{n} \quad(-1)^{n-i}\left(\begin{array}{c}
n \\
i
\end{array}\right)+f\left(x_{0}+\frac{n h}{2}\right)-f\left(x_{0}-\frac{n h}{2}\right) \\
\Rightarrow \Delta_{n}{ }^{s}\left(f, x_{0}, h\right)<f\left(x_{0}\right)(-1+1)^{n}+f\left(x_{0}+\frac{n h}{2} n h\right)-f\left(x_{0}-\frac{n h}{2}\right)
\end{gathered}
$$


$\Rightarrow \Delta_{n}{ }^{s}\left(f, x_{0}, h\right)<f\left(x_{0}+\frac{n h}{2}\right)-f\left(x_{0}-\frac{n h}{2}\right)<0$

Then $\quad S R D_{n} f \quad\left(x_{0}\right)=\lim _{h \rightarrow 0+} \frac{\Delta_{n}^{s}\left(f, x_{0}, h\right)}{h^{n}} \leq 0$, implies $x_{0} \in S$ and hence $y_{0} \in E, \quad$ a contradiction. So, our initial assumption is wrong. There can not be $\alpha$, $\beta \in[a, b]$, such that $\alpha<\beta$. So, non-decreasing in $[a, b]$.

$f(\alpha)>f(\beta)$. So, $f$ is

Theorem 3.4. Let $f$ be an upper semi-continuous function which has the property $D$ in $[a, b], S R D_{n} f$ $(x) \geq 0$ in in $[a, b]$ except an enumerable set $E$. Then $f$ is non-decreasing in $[a, b]$.

Proof. Suppose $\epsilon>0$ be arbitrarily small number

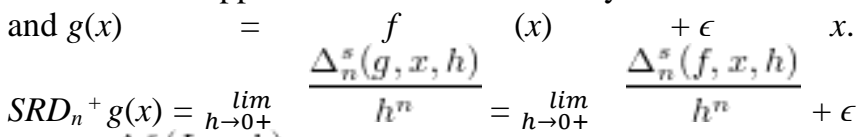
$\lim _{h \rightarrow 0+} \frac{\frac{\Delta_{n}^{s}(I, x, h)}{h^{n}}}{\text {, where } I(x)=x}$

$$
\Rightarrow S R D_{n}^{+} g(x) \leq S R D_{n}^{+} f(x)+\epsilon
$$

as $\Delta_{n}^{s}(I, x, h)=1$ if $n=1$ and $\Delta_{n}^{s}(I, x, h)=0$ if $n \geq 2$,

$$
\Rightarrow S R D_{n}^{+} g(x)=S R D_{n}^{+} f(x)
$$

Here, $g$ is also an upper semi-continuous function with property $D$ in $[a, b]$, moreover $g(E)$ is measurable thus contains no sub-interval. So, $g$ is non-decreasing in $[a, b]$. Since $\epsilon$ is arbitrarily small positive number, $f$ is nondecreasing in $[a, b]$.

Theorem 3.5. Let $f$ be an upper semi-continuous function which has the property $D$ in $[a, b], S R D_{n} f$ $(x) \geq 0$ almost everywhere in $[a, b], \operatorname{SRD}_{n}^{+} f(x)>-$ $\infty$ in $[a, b]$ except an enumerable set $E$. Then $f$ is nondecreasing in $[a, b]$.

\section{Proof. Let}

$A=\left\{x \in[a, b]: S R D_{n}^{+} f(x)<0\right\}$. Clearly, $m(A)=0$.

Suppose $\sigma$ is a continuous, non-decreasing function in $[a, b]$ such that $\Delta_{n}{ }^{s}(\sigma, x, h) \geq 0$ in $[a, b]$ except $A$. We consider an arbitrary small positive number $\epsilon$ and take $g(x)=f(x)+\epsilon \sigma(x)$. Then $g$ an upper semi-continuous function with property $D$ in $[a, b]$,

$S R D_{n}{ }^{+} g(x)$

$\lim _{h \rightarrow 0+} \frac{\Delta_{n}^{s}(g, x, h)}{h^{n}}$

$\lim _{h \rightarrow 0+}+\frac{\Delta_{n}^{s}(f, x, h)}{h^{n}}+\lim _{h \rightarrow 0+} \frac{\Delta_{n}^{s}(\sigma, x, h)}{h^{n}}$

$=S R D_{n}{ }^{+} f(x)+\epsilon \operatorname{SRD}_{n}{ }^{+} \sigma(x)$,

Therefore, $S R D_{n}{ }^{+} g(x) \geq 0$ almost everywhere in $[a, b]$ except $A$. Hence, $g$ is non-decreasing in $[a, b]$. Since $\epsilon$ is arbitrarily small positive number, $f$ is nondecreasing in $[a, b]$.

Note 3.6. Example of a function $\sigma$ which is continuous, non-decreasing in $[a, b]$ such that $\Delta^{n}(\sigma, \quad x, h) \geq 0$ in $[a, b]$ except a set $A$ of measure zero is a polynomial $a x^{k}+b x^{k-2}+\ldots+\lambda$, where the co-efficients are all positive and $k$ is an even natural number.

Theorem 3.7. If $f$ is continuous and $S R D_{n} f(x)$ exists in $[a, b]$ then $S R D_{n}^{+} f(x)$ has Darboux property in $[a, b]$.
Proof. Let us consider that $S R D_{n}^{+} f(x)$ does not have Darboux property, then there exist

$\alpha, \beta$ such that $f$ $(\alpha)<0, f(\beta)>0$ but $\operatorname{SRD}_{n}^{+} f(x) \neq 0$ for any $x \in(\alpha, \beta)$. Further, suppose $E^{+}=\left\{x \in[\alpha, \beta]: S R D_{n}^{+} f(x)>0\right\}, E^{-}$ $=\left\{x \in[\alpha, \beta]: S R D_{n}^{+} f(x)<0\right\}$, then

$[\alpha, \beta]=E^{+} \cup E^{-}$

Let $Q$ be (if any) non-degenerate component of $E^{+}$. Then $Q$ is an interval. Suppose $c, d$ be the end points of $Q$. $S R D_{n}{ }^{+} f>0$ in $Q$, so $f$ is non-decreasing in $Q$. $f$ being continuous and non-decreasing in $[c, d], S R D_{n}{ }^{+} f$ (c), $S R D_{n}{ }^{+} f(d)>0$. Therefore $c, d \in Q$, implies that $Q$ is a closed interval. $Q$ being arbitrary, every non-degenerate component of $E^{+}$is a closed interval. Following similar arguments, it can be shown that every non-degenerate component of $E^{-}$is a closed interval. Let $Q^{+}, Q^{-}$be the collection of all non-degenerate components of $E^{+}$and $E^{-}$respectively.Let $\mathbf{Q}=Q^{+} \cup Q^{-}$. Then any two distinct members of $Q$ are disjoint. Hence, $P=[\alpha, \beta]-\cup Q^{0}, Q \in \mathbf{Q}$, is perfect and $S R D_{n}{ }^{+} f$ has no point of continuity in $P$ relative to $P$, which is a contradiction $\quad$ as $S R D_{n}^{+} \quad f \quad \in B[\alpha, \beta]$. Therefore, $S R D_{n}{ }^{+} f(x)$ must have Darboux property.

Theorem 3.8. If $f$ is continuous in $[a, b]$ and $S R D_{n} f$ (x) exists in $(a, b)$ then there exists $c \in(a, b)$ such that $f$ (b) $-f(a)=(b-a) S R D_{n} f(c)$.

Proof. Here we may have following two cases Case 1:
Let $f$
(b)
$=f$
(a).
Then,

Subcase 1- In case $S R D_{n} f(x) \geq 0$ or $S R D_{n} f(x) \leq 0$ in $(a, b)$. Thus $f$ is monotone function. Now $f$ being continuous as well as monotone, $f$ is constant in $(a, b)$, ensuring the existence of $c$. Subcase 2-In case $f$ is not monotone, $S R D_{n} f(\alpha)<0$ and $S R D_{n} f(\beta)>0$ for some $\alpha, \operatorname{\beta in}(a, b)$ and hence there exists $\xi \in(a, b)$ such that $S R D_{n} f(\xi)=0$, implying $c=\xi$.

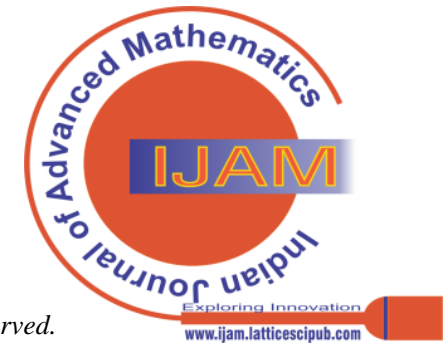




\section{Case 2:}

Let $f(b) \neq f(a)$. Then, suppose $\Phi(x)=f(x)-A x, A=$ constant. Clearly, $\Phi$ is continuous in $[a, b]$ and $S R D_{n} \Phi(x)$ exists in $(a, b)$.

$$
\text { Also, } S R D_{n} \Phi(x) \quad=S R D_{n} \quad f \quad(x) \text {. }
$$

Let us take $A=\frac{f(b)-f(a)}{b-a}$. Thus, $\Phi(b)=\Phi(a)$. By Case 1, there exists $c \in(a, b) \quad$ such that $S R D_{n} \Phi(c)=0$ $\Rightarrow S R D_{n} \quad f \quad(c) \quad=\frac{f(b)-f(a)}{b-a}$ This completes the proof of the theorem.

Note 3.9. Above results are applicable for any continuous function $f$.

\section{CONCLUSION}

From above analysis and discussion, it is clear that the symmetric Riemann-derivatives can be a new type of generalized derivatives, can follow many monotonicity, mean value property, Darboux property etc, like ordinary and some other derivatives but only if some conditions are satisfied. We have to work more to decrease the number of these conditions and to find more results on a more generalized derivatives.

\section{Application}

The above work on symmetric Riemann-derivatives provides scope of finding new derivatives of a function which are more generalized than ordinary derivatives, even some other generalized derivatives, under less number of restrictions. This work can provide clue for farther findings on Riemann fractional derivatives and can be used in differential equations, specially in electrical and mechanical phenomena analysis.

\section{ACKNOWLEDGMENT}

I am thankful to S. Ray (Department of Mathematics, Visva-Bharati University, India), A. Garai (Department of Mathematics, Memari College, India) and the mathematicians whose work [Refer to Reference] has motivated and guided me.

\section{DECLARATION}

There is no involvement of financial support and the work has no conflict of interest.

\section{REFERENCES}

1. Ash J. Marshall , "Generalizations of the Riemann derivative" , Trans. American Math. Soc. , 126, 1967, pp. 181 - 199. [CrossRef]

2. Ash J. Marshall, Catoiu Stefan and Chin William, "The classification of complex generalized Riemann derivatives" , Journal of Mathematical Analysis and Applications, 502(2), 2021. [CrossRef]

3. Belna C. L. , Evans M. J. and Humke P. D. , "Symmetric and ordinary differentiation" , Proc. American Math. Soc. , 72, 1978, pp. 261 - 267. [CrossRef]

4. Bertle R. G. and Sherbert D.R. , "Introduction to real analysis" , John Wiley Sons. , Inc. , New York, 1992.

5. Bruckner A.M. , "Differentiation of Real functions", Lecture Notes in Mathematics, Springer - Verlag, New York, 1978. [CrossRef]

6. Bullen P.S. and Mukhopadhyay S.N., "Relations between some general nth order derivatives" , Fund. Math. , 85, 1974, pp. 257 276. [CrossRef]

7. Butzer P. L. and Kozakiewicz W. , "On the Riemann derivatives for integrable functions" , Canad. J. Math. , 6, 1954, pp. 572 - 581 [CrossRef]
8. Subhankar Ghosh, "SOME STUDIES ON HIGHER ORDER GENERALIZED DERIVATIVES, SYMMETRIC DERIVATIVES, DIVIDED DIFFERENCES AND THEIR INTERRELATIONS' , Ph. D. Thesis, Visva-Bharati University, 2017, pp. 27 - 39.

9. Russel A. Gordon, "The Integrals of Lebesgue Denjoy, Perron, and Henstock", Graduate Studies in Mathematics, Volume 4, American Mathematical Society , 1994, pp. 121 - 136. [CrossRef]

10. Kundu N.K. , "On some conditions of symmetric derivatives implying monotonicity of functions", Rev. Roumaine Math. Pures Appl. , 15, 1970, pp. 561 - 568.

11. Kundu N.K. , "On some properties of symmetric derivatives", Ann. Polon Math. , 30, 1974, pp. 9 - 18. [CrossRef]

12. Mukhopadhyay S. N. , "Higher Order Derivatives", Chapman and Hall/CRC, Research monograph No 144, 2012. [CrossRef]

13. Radulescu Sorin, Alexandrescu Petrus and Alexandrescu DianaOlimpia, "Generalized Riemann Derivative" , Electronic Journal of Differential Equations, 74, 2013.

14. Radulescu Sorin, Alexandrescu Petrus and Alexandrescu DianaOlimpia, "The role of Riemann generalized derivative in the study of qualitative properties of functions" , Electronic Journal of Differential Equations, 187, 2013.

15. Thomson B.S., "Symmetric Properties of Real Functions", Marcel Dekker, Inc. , 1994

16. Zygmund A. , "Trigonometric Series, Vol I+II" , Cambridge University Press, 2002. [CrossRef]

\section{AUTHOR'S PROFILE}

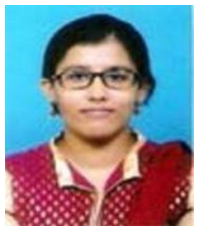

S. Deb, obtained M.Sc. in Pure Mathematics from University of Kalyani in 2014 and presently researching in Visva - Bharati, India on Generalized derivatives (Symmetric Riemann derivatives, Riemann-derivatives, Laplace Riemann-derivatives), trying to define new Generalized derivatives and new integrals using Borel derivatives similar to Perron integral and $Z_{n}$ integral. 